P126 (continued)

2.36, $\mathrm{p}=.05)$. More specifically, "lack of time" was higher (3.54 vs 2.79, $\mathrm{p}=.01$ ). Additionally, being "self-conscious about my looks when I do activities" was higher in the Reduce Phase ( 2.41 vs $1.58, \mathrm{p}=.007)$. No significant differences were found among benefits to PA $(4.52,4.36, \mathrm{p}=.45)$. Conclusions and Implications: While the Academy of Nutrition and Dietetics supports MRPs programs as an effective diet-related weight loss strategy, PA is traditionally not a focus. Health coaches could be used to facilitate MR program participants overcoming barriers to PA in the Reduce Phase while promoting noted benefits of PA in both the Reduce and Sustain Phases.

Funding: Sanford Health - South Dakota State University

\section{P127 Physical Activity and Sedentary Time Behaviors in Meal Replacement Program Participants}

Jessica Meendering, PhD, EP-C, Jessica.Meendering@

sdstate.edu, South Dakota State University, SIM 116C Box 2203, Brookings, SD 57007; H. Kleine, BS; A. Drooger, BS;

L. A. McCormack, PhD, MPH, RD, LN, EP-C;

K. Kattelmann, PhD, RDN, LN, FAND; S. Stluka, MS, RD, LN; J. Meendering, $P h D, E P-C$

Objective: To quantify physical activity (PA), sedentary time (ST) and stages of change for PA in meal replacement program (MRP) participants and evaluate if PA differs between MRP phase.

Design, Setting, and Participants: Twenty-four MRP participants (29-63y) in the Reduce (weight loss) Phase and 23 participants (31-82y) in the Sustain (weight maintenance) Phase were recruited for this cross-sectional study.

Outcome Measures and Analysis: PA and ST were assessed via accelerometer (G3TX+, ActiGraph LLC, Pensacola, FL) for 7 days. Average daily minutes of ST and PA were quantified in the following intensity categories: light (LPA), moderate (MPA), vigorous (VPA) and moderate-tovigorous (MVPA). Stage of Change (SOC) was assessed via the PA Stages of Change questionnaire. Linear regression was used to assess group (Reduce vs. Sustain) differences in PA while controlling for sex and age.

Results: Individuals in the Sustain Phase accumulated less ST (754.0 vs. $889.5, \mathrm{p}=.000)$ and greater LPA (291.5 vs. 182.0, $\mathrm{p}=.001)$, MPA (32.3 vs. 9.2, $\mathrm{p}=.000)$, VPA (3.1 vs. $0.25, \mathrm{p}=.028)$, and MVPA (35.4 vs. $9.5, \mathrm{p}=.000$ ) than individuals in the Reduce Phase. Only $20 \%$ of individuals in the Reduce Phase were meeting PA recommendations, compared to $70 \%$ of individuals in the Sustain Phase. $46 \%$ of Reduce Phase and $78 \%$ of Sustain Phase participants self-identified as being in the SOC maintenance phase.

Conclusions and Implications: The majority of participants in the Sustain Phase were meeting PA recommendations, which suggests the health coaching component may assist individuals in adopting more favorable PA behaviors as they transition through a MRP.

Funding: Sanford Health - South Dakota State University
P128 School-Based Obesity Prevention Policies and Practices and Unhealthy WeightControl Behaviors Among Adolescent Boys and Girls

Nicole Larson, PhD, MPH, RDN, larsonn@umn.edu, University of Minnesota, 1300 South Second Street, Suite 300, Minneapolis, MN 55454; C. S. Davey, MS; C. E. Caspi, ScD; M. Y. Kubik, PhD, MSN, RN; M. S. Nanney, PhD, MPH, RD

Objective: This study examines secular trends in secondary school curriculum topics relevant to the prevention of unhealthy weight-control behaviors; describes crosssectional associations between weight-related curriculum content and students' use of unhealthy weight-control behaviors; and assesses whether the implementation of school-based obesity prevention policies/practices are related to changes over time in students' behaviors.

Design and Participants: The Centers for Disease Control and Prevention Minnesota School Health Profiles and Minnesota Student Survey data were used along with National Center for Education Statistics data to examine secular trends $(\mathrm{n}=266$ schools); cross-sectional associations ( $\mathrm{n}=141$ schools); and longitudinal associations ( $\mathrm{n}=42$ schools).

Outcome Measures and Analysis: Students selfreported their height and weight and past-year use of unhealthy (fast or skip meals, smoke cigarettes) and extreme (use diet pills or other drugs, vomit on purpose, use laxatives) weight-control behaviors. Analyses accounted for school-level demographics.

Results: There was no observable pattern over the years 2002 to 2014 in the mean number of topics or prevalence of including topics addressing unhealthy weight-control behaviors in the curriculum. Prevalences in 2014 were $92.9 \%$ for risks of unhealthy weight-control practices, $92.1 \%$ for accepting body size differences, and $89.0 \%$ for eating disorders. The inclusion of all three topics versus $0-2$ topics and independently attention to eating disorders in the curriculum were inversely associated with schoollevel use of any extreme behaviors. In contrast, an overall measure of implementing school-based obesity prevention policies/practices (e.g., prohibited advertising) was unrelated to use of unhealthy or extreme behaviors.

Conclusions and Implications: Results suggest obesity prevention policies/practices do not have unintended consequences for student weight-control behaviors and support the importance of school-based health education as part of efforts to prevent unhealthy behaviors.

Funding: NIH

\section{P129 Determining If Differences in} Socioeconomic Status Are Associated With Screen Time Behavior and Physical Activity Levels

Noreen Syed, BS, nfsyed@uci.edu, University of California, Irvine, 1042 Mountain Grove Lane, Corona, CA 92881; M. Schneider, PhD 